\title{
Propuesta para la Reestructuración Funcional de la Figura del Coordinador de Virtualización en la UNED
}

\section{Ana María Martín-Cuadrado*1, Carlos Cerrada Somolinos², M. Angeles López- González ${ }^{3}$, Ignacio Quintana Frías ${ }^{4}$ y Mercedes García Pérez ${ }^{5}$}

\footnotetext{
1 Directora adjunta de Formación del Instituto Universitario de Educación a Distancia (IUED), Universidad Nacional de Educación a Distancia (UNED). Juan del Rosal, no 10, Madrid, España. amartin@iued.uned.es

2 Director del IUED, UNED. Juan del Rosal, nº 10, Madrid, España. director@iued.uned.es

3 Coordinadora de Redes de Investigación en Innovación Docente de la UNED. Juan del Rosal, no 10, Madrid, España. coordinacionredes@iued.uned.es

4 Unidad Técnica de Formación del IUED, UNED. Juan del Rosal, no 10, Madrid, España. i.quintana@iued.uned.es

5 Unidad Técnica de Formación del IUED, UNED. Juan del Rosal, no 10, Madrid, España. mgarcia@iued.uned.es
}

\section{Resumen}

El sistema organizativo académico de la Universidad Nacional de Educación a Distancia (UNED) está integrado por docentes en la Sede Central y profesores tutores en los Centros Asociados repartidos por el territorio español. A partir del nuevo panorama educativo en la enseñanza universitaria europea (Espacio Europeo de Enseñanza Superior, -EEES), la UNED se fue adaptando y situándose en un escenario moderno e innovador, iniciándose la mejora y homogeneización de los servicios que reciben los estudiantes mediante los cursos virtuales. En este contexto, se precisó la formación a profesores tutores y a estudiantes en la utilización de los cursos virtuales de la universidad, surgiendo así el rol del Coordinador de Virtualización (CV). En la actualidad hay 62 coordinadores (uno por cada centro asociado). Tras la implantación de las nuevas titulaciones dentro del marco del EEES y, una vez consolidada esta figura en la institución, se ha hecho necesario ofrecer una nueva organización en las funciones y tareas que los CV han de ejercer. Este trabajo describe la nueva propuesta organizativa diseñada para su aplicación a partir del próximo curso 2014-2015, y para cuya elaboración se han seguido los siguientes pasos: identificación del tipo de tareas a 
desarrollar (apoyo tecnológico a estudiantes y asesoramiento a profesores tutores), establecimiento de los contextos de trabajo (presencial y virtual), y clasificación por categorías para permitir una asignación de actividades básicas a todos los CV y de actividades más especializadas entre subgrupos de CV.

Palabras Ilave: Aprendizaje semipresencial; Formación de profesorado; Competencias tecnológicas; Habilidades digitales. 


\title{
Virtualization Coordinator: Trainer of Tutor-Professors and Support for Students in the UNED Associate Center
}

\begin{abstract}
The academic organizational structure of the Universidad Nacional de Educación a Distancia (UNED) is composed of professors at Central Headquarters and tutorprofessors at the Associate Centers spread across the Spanish territory. From the new educational landscape in European university education (European Higher Education Area - EHEA), UNED was adapting and placing itself in a modern and innovative stage, beginning with the improvement and standardization of services provided to students through the online courses. In this context, training for both tutor-professors and students in the use of online courses was required, and that's how the role of Virtualization Coordinator (VC) emerged. At present time, there are 66 VCs (one for each Associate Center). After implementation of the new qualifications within the framework of the EHEA and once established this role in the institution, it is necessary to provide a new organization in the functions and tasks that have to exercise VC. This paper describes a new organizational proposal designed for use from next year 20142015, and to whose development we have followed the following steps: identification of the type of tasks to be performed (technical support to students and advice to tutorprofessors), establishment of working contexts (presential and virtual), and categorization to allow distribution of basic activities among all the VC, and more specialized activities among subgroups of VC.
\end{abstract}

Keywords: Blended learning; Teachers training; Technological competences; Digital skills. 


\section{Introducción}

La UNED es la universidad de las grandes cifras: 250.000 estudiantes, 7000 profesores tutores, más de 1000 docentes, 62 centros asociados en la península y archipiélagos insulares agrupados en 9 campus virtuales, 18 centros en el extranjero, etc. En el modelo de formación de la UNED, blended learning o mixto, los equipos docentes necesitan apoyar el desempeño de su docencia en los profesores tutores para las tareas de seguimiento, apoyo y evaluación continua de los estudiantes (Quintana Frías, Martín-Cuadrado y Sánchez-Elvira, 2011). Los profesores tutores han venido ejerciendo sus funciones en los centros asociados y las tareas venían definidas por el ejercicio de la orientación y la tutoría a los estudiantes, según el Reglamento del Profesor-Tutor de la UNED (Junta de Gobierno el 13 de julio de 1990).

Con la incorporación al EEES, la diversificación de funciones del docente entre funciones docentes y funciones tutoriales ha cobrado una gran importancia, y conviene establecer el nivel de profundización que se requiere para llevar a cabo las tareas; en el caso de la UNED es especial por la casuística de compartir funciones entre varias figuras (Martín-Cuadrado et al., 2012).

Con la inclusión y expansión de las Tecnologías de la Información y Comunicación (TIC) en la educación a distancia, el contexto de aprendizaje virtual ha variado, potenciando las posibilidades para enseñar y aprender, por lo que el rol del docente y del tutor se ha replanteado y asistimos, en la actualidad, a una eclosión de escenarios de aprendizaje que suponen otras formas de enseñar, tutorizar y evaluar al estudiante. En la UNED, el profesor tutor alterna las tareas de orientación, seguimiento y evaluación en diferentes escenarios: centros asociados y escenario presencial; plataforma AVIP y escenario semipresencial, y plataforma aLF y escenario virtual. En los Estatutos de la UNED, aprobados en 2005, así se establece: "Los profesores-tutores realizarán la actividad tutorial presencial en los centros asociados y utilizarán los medios tecnológicos de comunicación que la UNED adopte en su modelo educativo" (Art.141, Apdo. 2). En la actualidad, el profesor tutor adquiere diversas denominaciones de acuerdo al contexto de intervención: profesor tutor de centro asociado, profesor tutor campus o profesor tutor intercampus. De acuerdo al contexto, las funciones y tareas son diversas y puntuales (Tabla 1). 
Tabla 1: Modalidades de Tutoría en los Grados. UNED.

\section{Modalidades de \\ Tutoría \\ Características}

Dónde se desarrolla En tu Centro Asociado y con qué medios

\section{Tutoría de Centro Tutoría de Campus}

\begin{tabular}{llll}
\hline Asignaturas & $\begin{array}{l}\text { Curso de Acceso } \\
\text { mayores } 25 \text { y } 45 \text { años }\end{array}$ & $\begin{array}{l}1^{\circ} \text { a } 40 \text { curso } \\
\text { (asignaturas con más } \\
\text { de } 400 \text { estudiantes). }\end{array}$ & $\begin{array}{l}3^{\circ} \text { y } 4^{\circ} \text { curso } \\
\text { (asignaturas con } \\
\text { menos de } 400 \\
\text { estudiantes) }\end{array}$
\end{tabular}

\begin{tabular}{llll}
\hline Estudiantes: & $\begin{array}{l}\text { A criterio del Centro } \\
\text { Asociado }\end{array}$ & $\begin{array}{l}\text { A criterio de los } \\
\text { Centros Asociados del } \\
\text { Campus. }\end{array}$ & $\begin{array}{l}\text { A partir de cuarenta } \\
\text { estudiantes }\end{array}$ \\
No Grupos & $\begin{array}{l}1 \text { grupo/Centro } \\
\text { Asociado }\end{array}$ & $\begin{array}{l}\text { Centros Asociados del } \\
\text { Campus }\end{array}$ & $\begin{array}{l}\text { Varios grupos/Centros } \\
\text { Asociados de todos } \\
\text { los Campus }\end{array}$ \\
\end{tabular}

\begin{tabular}{ll}
\hline $\begin{array}{l}\text { Duración de la } \\
\text { tutoría/ }\end{array}$ & $50-60^{\prime}$ \\
Grabación & No \\
\hline $\begin{array}{l}\text { Profesor Tutor: } \\
\text { funciones }\end{array}$ & $\begin{array}{l}\text { Sesiones de tutoría } \\
\text { semanales }\end{array}$ \\
\hline
\end{tabular}

Facilita orientaciones para la preparación de la asignatura

Aclara dudas de contenidos

Realiza actividades prácticas

Explica los criterios aplicados en la corrección de las PEC

Orienta para las pruebas presenciales.

En tu Centro
Asociado/ Aula AVIP o
en tu ordenador

En tu Centro en tu ordenador

\section{Tutoría Intercampus}

A través de tu ordenador, mediante videoconferencia desde el curso virtual

$50-60^{\prime} \quad 50-60$

\section{Opcional Si}

\section{Sesiones de tutoría Sesiones de tutoría semanales (grabación \\ semanales (grabación)} opcional)

Facilita orientaciones Facilita orientaciones para la preparación de la asignatura

Aclara dudas de contenidos

Realiza actividades prácticas

Explica los criterios aplicados en la corrección de las PEC

Orienta para las pruebas presenciales. para la preparación de la asignatura

Aclara dudas de contenidos

Realiza actividades prácticas

$$
\begin{aligned}
& \text { Explica los criterios } \\
& \text { aplicados en la } \\
& \text { corrección de las PEC } \\
& \text { Orienta para las } \\
& \text { pruebas presenciales. }
\end{aligned}
$$




\section{Seguimiento del curso virtual}

Facilita información en el foro de tutoría sobre las actividades que va a llevar a cabo en la tutoría semanal

\section{Seguimiento del curso virtual}

Facilita información en el foro de tutoría sobre las actividades que va a llevar a cabo en la tutoría semanal
Participación en la evaluación continua de las PEC

\author{
Participación en la \\ evaluación continua \\ de las PEC
}

\section{Seguimiento del curso virtual}

Atención en los foros generales de las posibles dudas sobre los contenidos de las sesiones de tutoría que haya realizado.

En el foro del grupo de tutoría mantiene contacto con los estudiantes de cuya evaluación continua se ocupa.

\section{Participación en la evaluación continua de las PEC}

\section{La figura del Coordinador de Virtualización}

EI IUED de la UNED acometió un plan de formación de profesores tutores para que pudieran asumir funciones y tareas de acuerdo a su incorporación en los Grados, y de forma complementaria, para capacitarles tecnológicamente. La estructura que se utiliza es la de formación en cascada (Aguiar, Santamaría Lancho y Sánchez-Elvira Paniagua, 2008), mejorando los sistemas de formación empleados en los sistemas a distancia y favoreciendo el seguimiento y apoyo a los tutores en la comunicación con sus estudiantes.

Para llevar a cabo este cometido, se contó con la Unidad Técnica de Formación del IUED y con el Coordinador de Virtualización, profesor tutor ubicado en cada uno de los centros asociados distribuidos en los nueve campus. Al CV se le considera integrante de la red de formadores para el uso de las TIC de la UNED, instruidos previamente en los mismos contenidos y competencias tecnológicas requeridos para la formación y evaluación posteriores de sus tutores (Quintana Frías et al., 2011; MartínCuadrado et al., 2012). 
El Consejo de Gobierno de la UNED, en su reunión del día 28 de junio de 2006, aprobó en el punto 7, Anexo XXXIII (ref. http://portal.uned.es/archivos/ActasCG/CG 2006 11 28.pdf) y a solicitud del Vicerrectorado de Calidad e Innovación Docente una "propuesta para la mejora y homogeneización de los servicios que reciben los estudiantes mediante los cursos virtuales" (ref. http://www.uned.es/bici/curso2005-2006/060904/38-1.html\#1).

Se establecía la figura del Coordinador de Virtualización (CV), que nombrado por el Director del Centro Asociado, dependería funcionalmente del IUED. La figura del CV surgió para responder a la necesidad de formar a los profesores tutores en la utilización de los Cursos Virtuales, entre otras cosas (Martín-Cuadrado et al., 2012).

El CV es el nexo de unión entre la Sede Central y los Centros Asociados, unos de los factores clave para la expansión telemática y la incorporación de las TIC (Aguiar, Sánchez-Elvira Paniagua y Martín-Cuadrado, 2012).

\section{Identificación y análisis de tareas previas de los Coordinadores de Virtualización}

De cara a la elaboración de una nueva propuesta de asignación de actividades a los CV ha sido necesario en primer lugar estudiar el tipo de tareas que venían ejerciendo. Con ellos se ha pretendido identificar aquéllas que están vigentes en la actualidad, aquellas otras que se han quedado obsoletas ( $y$ por tanto se pueden eliminar), así como las que se pueden considerar demasiado especializadas y que pueden ser llevadas a cabo por un subgrupo más reducido de CV.

Así, las funciones y tareas que han venido desarrollando los CV hasta el curso 2013-2014 se pueden ver en la siguiente tabla (Tabla 2). 
Tabla 2: Funciones y tareas del Coordinadores de Virtualización.

\section{Funciones y tareas de los CV}

- Información a los profesores tutores sobre los usos y recursos de CiberUNED.

- Formación de profesores tutores en el uso de la plataforma WebCT -Tutor-T, II y II(hasta el curso 2008-2009).

- Formación en línea de los profesores tutores para el EEES. Supone el entrenamiento de los tutores en el uso de la plataforma de formación del curso (aLF). Nivel I y II. Desde el curso 2008-2009.

- Formación de los profesores tutores de apoyo en red (TAR) del Centro Asociado para el uso de las herramientas de administración de webCT y de aLF.

- Colaborar en la formación y desarrollo profesional de los profesores tutores en modalidad en línea.

- Diseño y elaboración de recursos para facilitar la formación y desarrollo profesional de los profesores tutores.

- Apoyo a las acciones del Plan de Acogida relacionadas con la utilización de las tecnologías:

- Información a los estudiantes sobre el uso de los recursos que ofrecen los cursos virtuales.

- Seminarios presénciales sobre los cursos virtuales (se proponen 3 anuales, al menos).

- Diseño y elaboración de recursos y materiales para los seminarios.

- Talleres sobre el uso de herramientas tecnológicas (podrían colaborar con el desarrollo de estos talleres en el marco de los cursos de acogida de los CCAA).

- Atención presencial a los estudiantes con dudas.

- Apoyo en el curso e_UNED primeros pasos.

- Al margen de sus funciones, los CVs podrán participar en acciones formativas del IUED realizadas en línea, en este caso percibirían las remuneraciones complementarias, para el desempeño de esa tarea (por ejemplo, el curso Formación Inicial de Tutores -FIT-).

Por otro lado, realizando un análisis de la evolución que han experimentado las enseñanzas del EEES en la UNED desde su implantación hasta la actualidad (20132014) se observa que:

- Se han implementado los grados y postgrados EEES, se ha conseguido el ordenamiento de la UNED en nueve campus. 
- Se han incorporado otras figuras en los centros asociados, como el Coordinador Tecnológico (complemento técnico a la figura del CV, más formativa).

- Se han consolidado figuras como el Tutor de Apoyo en Red (TAR de grados y postgrados).

- Han aparecido figuras tutoriales de apoyo en los postgrados (profesores tutores virtuales), y otras en los grados (tutor campus y tutor intercampus).

- Se han generado comunidades virtuales de acogida y programas de formación para estudiantes nuevos, etc.

A partir de estas evidencias se constata la realidad de la UNED en el momento actual y se pone de manifiesto la necesidad de reconducir la figura del CV y las funciones y tareas que realiza. Como solución se plantea la elaboración de una nueva propuesta que permita:

- Visibilizar en la comunidad universitaria la red de formadores del IUED ubicados en los centros asociados.

- Asegurar la sostenibilidad del CV en la UNED.

\section{Propuesta de las funciones y tareas de los Coordinadores de Virtualización (2014-2015)}

En primer lugar conviene hacer una reflexión sobre la localización actual de los CV de que dispone la UNED. Los 66 CV se sitúan en los Centros Asociados, tal como se muestra en la Figura 1. 


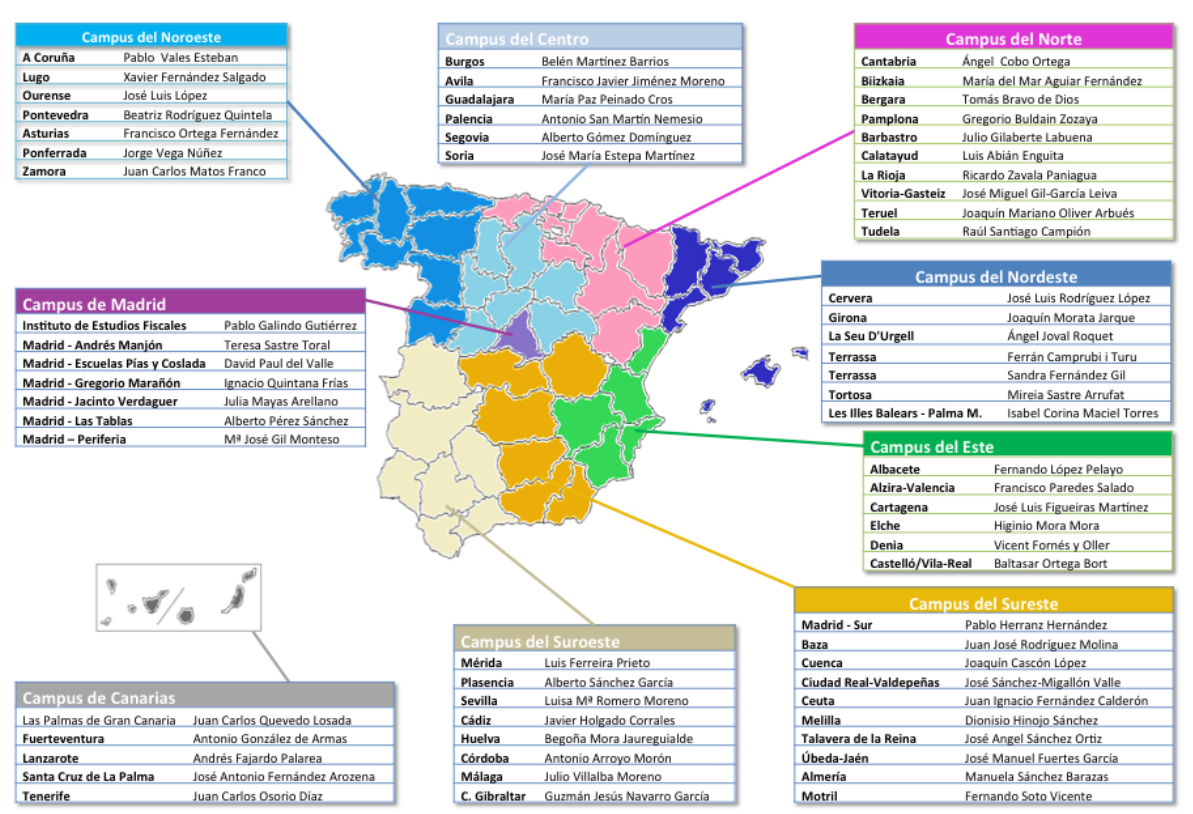

Figura 1. Coordinadores de Virtualización en los Centros Asociados de la UNED.

Fuente: Imagen de los propios autores.

Los CV podrían realizar funciones y tareas en dos contextos de intervención, en el Centro Asociado (entorno presencial) y en línea (entorno virtual):

- Tareas presenciales, se llevarán a cabo en el centro asociado específicamente.

- Tareas virtuales, se realizarán actividades bien en el campus de procedencia o bien actividades intercampus.

El ordenamiento y estructuración de las funciones y tareas respecto a cursos anteriores se ha editado $y$, en este momento hemos comprobado que hay tareas que tienden a desaparecer o son más livianas (ej. Curso de formación EEES; desde el año 2009 se han realizado 15 ediciones, y se ha formado a 6937 profesores tutores); han aparecido otras tareas o han tomado más fuerza (ej. Participación en el Plan de Acogida de los estudiantes nuevos, que implica la realización de Seminarios Tecnológicos de Acogida, el curso e_UNED Primeros pasos, etc.). Con todo ello, el panorama ha cambiado y creemos que debemos seguir respondiendo a los retos que plantea la universidad y que sean de la competencia del IUED.

Las tareas en el contexto presencial, clasificadas por cuatrimestres y de acuerdo al colectivo de atención (profesor tutor/estudiante), se recogen en la Tabla 3. 
Tabla 3: Tareas del CV. Contexto presencial.

\begin{tabular}{|c|c|c|c|}
\hline Tareas & $10 \mathrm{C}$. & $2^{\circ} \mathrm{C}$. & $\mathbf{C P}$ \\
\hline \multicolumn{4}{|l|}{ PROFESORES TUTORES } \\
\hline Información/formación Campus UNED/aLF & $*$ & & * \\
\hline Comunidad alF Centro Asociado & $*$ & $*$ & $*$ \\
\hline Acogida de PT en FIT & $*$ & & $*$ \\
\hline Curso EEES (dinamización, seguimiento y evaluación) & $*$ & $*$ & * AP \\
\hline Desarrollo profesional PT (presencial) & $*$ & $*$ & * AD \\
\hline Formación TAR grados y postgrados: acogida, inicial y desarrollo & * & $*$ & * AP \\
\hline
\end{tabular}

\section{ESTUDIANTES}

Apoyo Jornada Acogida CC.AA.

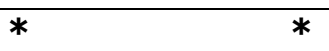

Seminarios Campus UNED/curso virtual

Otros seminarios competencias TIC

Nota. C (Cuatrimestre); CP (Contexto Presencial); AP (Apoyo); AD (A Demanda).

Las tareas en el contexto virtual, clasificadas por cuatrimestres y de acuerdo al colectivo de atención (profesor tutor, estudiante, docentes y otros), aparecen en la Tabla 4. 
Tabla 4: Tareas del CV. Contexto virtual.

Tareas

\section{Profesores tutores}

Información/formación Campus UNED/aLF.

FIT (Coordinación, seguimiento y evaluación)

Curso EEES (coordinación)

Curso EEES (dinamización, seguimiento y evaluación)

Desarrollo profesional PT (en línea, en abierto)

Elaboración y diseño de recursos y materiales para el aprendizaje autónomo de los PT.

Formación CV: acogida, inicial y desarrollo

Formación TAR grados y postgrados: acogida, inicial y desarrollo

Formación PT postgrados: acogida, inicial y desarrollo

Formación PT intercampus: acogida, inicial y desarrollo

Formación PT CUID

$1^{\circ} \mathrm{C} . \quad 2^{\circ} \mathrm{C} . \quad \mathrm{CL}$

$*$

* IUED

* * IUED

* $\quad * \quad *$

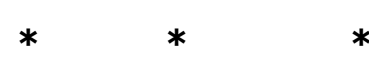

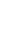

$*$

QM

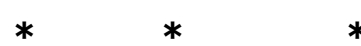

$* \quad * \quad *$

$* \quad * \quad *$

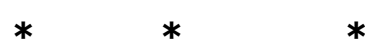

\section{Estudiantes}

E_UNED (coordinación)

E-UNED (apoyo)

Comunidad Acogida Estudiantes Doctorado

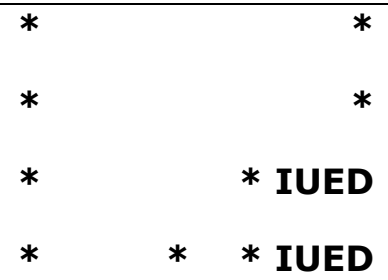

Comunidad Acogida Estudiantes Movilidad

PDI

Actividades formativas en línea

Actividades formativas en abierto

\section{Otros}

Coordinación Comunidad CV

Coordinación Comunidad TAR

Coordinación Comunidad Intercampus

$* \quad * \quad$ IUED

Dinamización Comunidades PT

Nota. C (Cuatrimestre); CL (Contexto en Línea); QM (en Cualquier Momento). 
La diversificación de tareas, de acuerdo al contexto, implicará un seguimiento por parte del IUED a través de la Comunidad Virtual de CV.

La mayoría de las tareas que exijan la participación de un grupo de CV serán coordinadas por un CV, apoyado por el IUED. Se establecerá un subgrupo de trabajo en la Comunidad Virtual de CV que facilitará la comunicación y el intercambio de información entre el coordinador y su equipo, evitando ruidos entre los CV que no estén participando.

El IUED podrá seleccionar al CV idóneo para el desempeño de las tareas en el contexto virtual. Para poder realizar esta selección con un criterio válido y transparente, se ha diseñado un formulario que recogerá la candidatura del CV.

Las tareas realizadas en el contexto presencial son las mismas para todos los CV. Se pretende asegurar un mínimo de actuaciones similares, dejando otras actividades a criterio del centro asociado. Entre las tareas relacionadas con el Plan de Acogida para estudiantes nuevos, el IUED-Formación se compromete en la orientación para la realización y evaluación de los Seminarios Tecnológicos de Acogida a través de un cuestionario disponible en línea. Este cuestionario está diseñado para valorar aspectos relacionados con datos sociodemográficos (tanto personales como académicos), diversas preguntas sobre la satisfacción con el seminario y una pregunta sobre las posibles necesidades de formación.

Por cada tarea realizada se demandará un informe-memoria que asegure el cumplimiento exacto de la tarea y nos aporte un feed-back sobre el diseño e implementación de la tarea. En el caso de las tareas realizadas en el contexto presencial, el informe-memoria vendrá con el Vo Bo de la dirección del centro asociado. El informe-memoria se realizará y entregará en línea.

\section{Conclusiones}

En este trabajo se ha presentado una propuesta de reorganización de las funciones y tareas que hasta el momento venía desarrollando en la UNED una figura clave como es el Coordinador de Virtualización. La propuesta es fruto de una reflexión profunda sobre la situación actual de la implantación de las enseñanzas del EEES en la 
UNED que ha provocado una remodelación obligada. En el momento actual el modelo presentado ha sido aprobado y se ha decidido implantar a lo largo del curso 20142015, por lo que no se pueden aportar datos específicos de su aplicación.

En todo caso podemos indicar que el CV ha sido una figura de formación y apoyo a profesores tutores y estudiantes y que con el nuevo modelo seguirá siéndolo. Su grado de visibilidad ha sido diferente en cada Centro Asociado dependido, mayoritariamente, de su implicación en las tareas y de la posterior evaluación por parte del IUED. Con el nuevo modelo pretendemos que el CV sea una figura clave visible en el Centro Asociado y en la UNED, para lo que se ha diseñado un sistema eficaz de recogida de información sobre las actividades realizadas. Así mismo, pretendemos potenciar su utilidad como formador de formadores, y maximizar el recurso de cara a los estudiantes.

Por último, pensamos que la coordinación del CV con el resto de coordinadores (Académico, Tecnológico, Extensión Universitaria y COIE) en cuanto a, por ejemplo, oferta y apertura de actividades que impliquen a otros actores del territorio donde se ubica el centro asociado, podría aportar mayor riqueza cultural, social y económica a estas sedes geográficas.

\section{Referencias}

Aguiar-Fernández, M. M., Sánchez-Elvira-Paniagua, A., \& Martín-Cuadrado, A. M. (2012). La preparación de los profesores tutores de la UNED: de la presencialidad a la virtualización. Revista Iberoamericana para la Investigación y el Desarrollo Educativo, 8, 1-17.

Aguiar-Fernández, M. M., Santamaría Lancho, M., \& Sánchez-Elvira-Paniagua, A. (2008, julio). ¿Cómo preparar a los tutores presenciales para la tutoría en línea? El caso de la UNED. Trabajo presentado en el IX Encuentro Internacional Virtual Educa, Zaragoza, España.

Quintana Frías, I., Martín-Cuadrado, A. M., \& Sánchez-Elvira, A. (2011, julio). Programa de formación en línea para la incorporación de los profesores tutores de la UNED a las titulaciones de Grado. Comunicación presentada en las VIII 
Jornadas Internacionales de Innovación Universitaria. Retos y oportunidades de los nuevos títulos en educación superior. Universidad Europea de Madrid, Madrid, España.

Martín-Cuadrado, A. M., Sánchez-Elvira, A., Luzón Encabo, J.M., Quintana Frías, I., Aguiar Fernández, M. Mar., Marauri Martínez de Rituerto, P., \& Santamaría Lancho, M. (2012). El plan de adaptación de los tutores al EEES: Balance de tres cursos académicos. Comunicación presentada en el I Congreso Internacional de la Red Iberoamericana de Investigación sobre la Calidad de la Educación Superior (RIAICES). Universidad La Salle. Canoas. Brasil. 